\title{
Visually Striking and Alarming Phenomenon - Case Report of Purple Urine Bag
}

\section{Syndrome}

\section{Priya Patel*}

*Correspondence: Priya Patel

Address: Internal Medicine Residency, Florida Atlantic University, Boca Raton, Florida

e-mail $\bowtie$ : ppatel65@health.fau.edu

Received: 17 September 2021; Accepted: 27 September 2021

Copyright: (C) 2021 Priya P. This is an open-access article distributed under the terms of the Creative Commons Attribution License, which permits unrestricted use, distribution, and reproduction in any medium, provided that the original work is properly cited.

\section{ABSTRACT}

Purple urine bag syndrome has been identified since 1978 with higher prevalence among those individuals who reside in nursing homes and those with a long-term indwelling Foley catheter. In addition to prolonged catheterization and institutionalization, the female gender, alkaline urine, and chronic constipation are other predisposing factors. Purple urine bag syndrome is also associated with bacterial urinary tract infections that produce enzymes, sulphatase and phosphatase involved in the breakdown of tryptophan. The final metabolites include indigo, which has a blue hue, and indirubin with a red hue, which when mixed, produce a purple color. Treatment includes antibiotics for the associated urinary tract infection as well as exchange of the Foley catheter and appropriate catheter care using proper sterile technique. However, there are no set guidelines instructing how aggressively to treat. The patient is a 60 -year-old male who presented to the hospital for clogged chronic Foley catheter who developed purple urine bag syndrome.

Keywords: Purple Urine, Catheter Associated Urinary Tract Infection, Tryptophan, Sulphatase, Phosphatase, Escherichia Coli, Provedencia Stuartii

\section{Introduction}

Purple urine bag syndrome has been noted to occur predominantly in people chronically debilitated necessitating residence in a nursing home or rehabilitation facility (Su et al., 2005) as well as individuals with chronic Foley catheter placement (Lin et al., 2008). Ocular diagnosis of purple urine bag syndrome at times can be delayed or missed if the provider is unaware of such occurrence. The diagnosis can be delayed as there are other confounders that cause urine color change including hematuria, methylene blue, food dyes, rhabdomyolysis, nephrolithiasis, benign prosthetic hyperplasia, malignancy, poisons and porphyria (Agapakis et al., 2014). The site can be quite alarming and worrisome, but recognition is important as the treatment is quite simple.

\section{Case Presentation}

A 60-year-old male with history of diabetes mellitus type II, hypertension, hyperlipidemia and non- 
ischemic cardiomyopathy with automatic implantable cardioverter- defibrillator (AICD) placement who presented to the hospital for a clogged Foley catheter associated with distended lower abdomen, diaphoresis and sinus tachycardia. The initial Foley catheter was placed during the patient's previous three-month hospital stay to keep urine from entering his sacral stage III decubitus ulcer. When he was discharged from the hospital to the rehabilitation facility, the Foley catheter remained, and it was exchanged two times at the facility where he has been residing for three months.

He was admitted to the hospital for a catheter associated urinary tract infection and his Foley catheter was exchanged in the emergency department. Initially the Foley catheter was draining yellow, purulent urine with sediment and he was started on broad spectrum antibiotics. His urine analysis was significant for $\mathrm{pH}$ of 8 .

The following day, when he was being evaluated, his urine was noted to be a lavender purple color in the Foley bag (Fig. 1) and his urine culture was growing extended spectrum beta-lactamase Escherichia coli and Provedencia stuartii for which he was initiated on appropriate antibiotics.

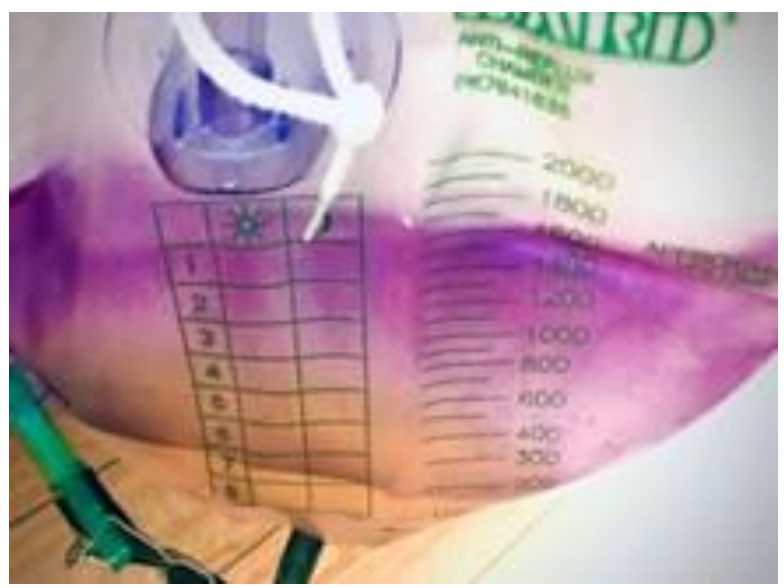

Figure 1: Purple urine collection in Foley bag.

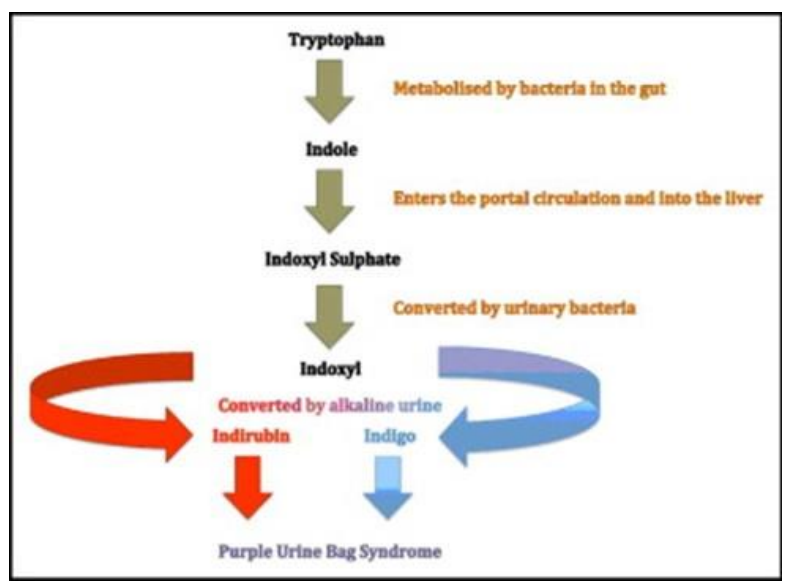

Figure 2: Tryptophan metabolism (Al-Sardar and Haroon, 2009). 


\section{Discussion}

Purple urine bag syndrome has been identified since 1978 with higher prevalence among those individuals who reside in nursing homes and require a long-term indwelling Foley catheter with rates of occurrence as high as 9.8\% (Khan et al., 2011). The population most commonly affected includes people of the female gender, those with alkaline urine, chronic constipation, individuals requiring institutionalization and those requiring the use of plastic urinary catheter and bag (Su et al., 2005; Lin et al., 2008).

The etiology of this purple urine phenomenon has been suggested as a series of events starting with the metabolism of dietary tryptophan by enteric bacteria producing indole (Stott et al., 1987). Indole gets absorbed in the portal circulation and is converted in the liver to indoxyl sulphate. Now, the indoxyl sulphate gets excreted in the urine and bacteria producing sulphatase and phosphatase enzymes convert indoxyl sulphate to indoxyl, which is then broken down into indigo (blue) and indirubin (red) as evidenced in Fig. 2. When these metabolites are mixed, they produce purple urine. Examples of organisms that are responsible for this magnificent site include Pseudomonas aeruginosa, Proteus mirabilis, Morganella morganii, Escherichia coli, Provedencia stuartii and rettgerri (Lin et al., 2008).

The interesting part about purple urine bag syndrome is that not all bacteria of the same species produce the enzymes sulphatase and phosphatase (Dealler et al., 1988). Another factor that plays a role is an alkaline environment as well the material used in production and manufacturing of the Foley catheter and collecting urine bag (Jones et al., 2003).

Purple urine production can occur within hours to days after placement of an indwelling catheter. In the case of this patient, he told us that he had two instances of the purple urine while he was at the rehabilitation facility and overtime the urine cleared after Foley catheter exchange (Hsuan et al., 2002).

In a study conducted by $\mathrm{Su}$, et al. (2005) a total of 157 patients with urinary catheters were studied, of which 13 exhibited purple urine bag syndrome. These 13 individuals were analyzed based on several factors including age, functional status, duration of catheterization, number of daily medications, living location, feeding route, bowel habits, and the pattern of use of a urinary catheter. They found that 9 out of the 13 patients lived in a nursing home and 11 out of the 13 individuals had a Foley catheter. The $\mathrm{pH}$ for 12 out of 13 purple urine bag syndrome patients' urine was > or = 7 (Su et al., 2005). In reviewing this study, it brings light to this patient who had decreased functional capacity after being hospitalized for three months during which time he developed a sacral stage III decubitus ulcer with subsequent Foley catheter placement in order to prevent urine from entering and infecting the decubitus ulcer. 
The treatment of purple urine bag syndrome involves usage of appropriate antibiotics, whether it be intravenous for immunocompromised patients or oral antibiotics along with exchange of the Foley catheter to prevent recurrent urinary tract infections (Agapakis et al., 2014). There have been some reported cases of persistent purple urine bag syndrome causing Fournier's gangrene requiring urological intervention and debridement (Khan et al., 2011).

It seems that there needs to be a perfect series of events in order to develop purple urine bag syndrome including increased dietary tryptophan along with the presence of a chronic indwelling catheter and a sulphatase and phosphate bacteria. In 2011, catheter associated urinary tract infections (CAUTI) was about two per 1000 people in the United States but despite the common occurrence of CAUTI, purple urine bag syndrome remains a rare phenomenon [1].

\section{Conclusion}

Purple urine bag syndrome itself is a benign and harmless condition, however efforts should be focused on minimizing prolonged catheterization and attempts to promptly remove Foley catheters when the individual no longer requires usage to reduce the incidence of catheter associated urinary tract infections. Among individuals that reside in nursing homes, particularly our elderly who may require chronic indwelling catheterization, efforts should be made to improve catheter care.

\section{References}

Agapakis D, Massa E, Hantzis I, Paschoni E, Satsoglou E. Purple Urine Bag Syndrome: a case report of an alarming phenomenon. Hippokratia 2014; 18: 92-94.

Al-Sardar H and Haroon D. Purple urinary bag syndrome. Am J Med 2009; 122: e1-2.

Dealler SF, Hawkey PM, Millar MR. Enzymatic degradation of urinary indoxyl sulfate by Providencia stuartii and Klebsiella pneumoniae causes the purple urine bag syndrome. J Clin Microbiol 1988; 26 : 2152-2156.

Hsuan-Hwai Lin, Sheng-Jiun Li, Kao-Bin Su, and Lian-Shan Wu. Purple Urine Bag Syndrome : A Case Report and Review of the Literature. J Intern Med Taiwan 2002; 13: 209-212

Jones RA, Deacon HJ, Allen SC. Two cases and a short discussion of purple urine bag syndrome. $C M E$ Journal Geriatric Medicine 2003; 5: 84-87.

Khan F, Chaudhry MA, Qureshi N, Cowley B. Purple urine bag syndrome: an alarming hue? A brief review of the literature. Int J Nephrol 2011; 2011: 419213.

Lin $\mathrm{CH}$, Huang HT, Chien CC, Tzeng DS, Lung FW. Purple urine bag syndrome in nursing homes: ten elderly case reports and a literature review. Clin Interv Aging 2008; 3: 729-734. 
Stott A, Khan M, Roberts C, Galpin IJ. Purple urine bag syndrome. Ann Clin Biochem 1987; 24: 185188.

Su FH, Chung SY, Chen MH, Sheng ML, Chen CH, Chen YJ, Chang WC, Wang LY, Sung KY. Case analysis of purple urine-bag syndrome at a long-term care service in a community hospital. Chang Gung Med J 2005; 28: 636-642.

1. National healthcare quality and disparities report- chartbook on patient safety. [Jun;2019]; 1. Comstock. CHARTBOOK ON. https://www.ahrq.gov/sites/default/files/wysiwyg/research/findings/nhqrdr/chartbooks/patien tsafety/qdrpatientsafetychartbook-2017update-090617.pdf 2017 